\title{
Motor Cortical Plasticity Induced by Volitional Muscle Activity-Triggered Transcranial Magnetic Stimulation and Median Nerve Stimulation
}

Pramudika Nirmani Kariyawasam

Health Sciences University of Hokkaido

Shinya Suzuki

Health Sciences University of Hokkaido

Susumu Yoshida ( $\square$ ysdssm@hoku-iryo-u.ac.jp )

Health Sciences University of Hokkaido

\section{Research}

Keywords: Bilateral training, Transcranial magnetic stimulation, functional electrical stimulation, neuroplasticity

Posted Date: October 13th, 2020

DOI: https://doi.org/10.21203/rs.3.rs-89368/v1

License: (c) (i) This work is licensed under a Creative Commons Attribution 4.0 International License. Read Full License

Version of Record: A version of this preprint was published at Brain Sciences on December 31st, 2021. See the published version at https://doi.org/10.3390/brainsci12010061. 


\section{Abstract}

\section{Background}

Bilateral motor training (BMT) is a useful method to modify the excitability of the corticospinal system. The effects of artificial symmetrical movement on corticospinal excitability through functional electrical stimulation (FES) or transcranial magnetic stimulation (TMS) have not been reported. Therefore, we compared motor-evoked potentials (MEPs) following TMS over the ipsilateral primary motor cortex (M1) of voluntary movements after conventional BMT and repetitive artificial symmetrical movements generated through FES and TMS.

Methods

Surface electromyograms of the abductor pollicis brevis (APB) muscles were recorded bilaterally in 12 healthy participants. Three sessions with different protocols were conducted: (1) bilateral finger training (BFT) involving bilateral thumb abduction, (2) right APB-triggered TMS of the ipsilateral M1 (APBtriggered i-TMS), and (3) right APB-triggered contralateral median nerve stimulation (APB-triggered cMNS). Each protocol consisted of 360 trials for 30 min. Resting motor threshold (RMT), MEPs induced by single-pulse TMS, short-interval intracortical inhibition (SICI), and intracortical facilitation (ICF) induced by paired-pulse TMS were assessed as outcome measures at baseline and at $0,20,40$, and 60 min after intervention.

Results

RMT showed no significant effect of intervention, time, or interaction. MEP amplitude showed a significant effect with time. MEP amplitude significantly increased at 0, 20, and 40 min post-intervention in BFT; at 0, 20, 40, and 60 min post-intervention in APB-triggered i-TMS; and at 20 and 40 min postintervention in APB-triggered c-MNS in comparison to the baseline values. SICl was significantly decreased at 0 min post-intervention in the BFT and APB-triggered i-TMS protocols. ICF was significantly increased at 0 min post-intervention in the BFT and at 20 min post-intervention in the APB-triggered cMNS protocol.

Conclusion

The main finding of the present study was the long-lasting increase in MEP amplitude in all three mirrorsymmetrical movement protocols. The observed changes are long-lasting and comparatively strong. However, the underlying neural mechanisms seem to be slightly different across the three protocols. Thus, whether voluntarily or artificially caused, repetitive symmetrical mirror movements enhance corticospinal excitability.

\section{Background}


Bilateral motor training (BMT) is an effective method to improve upper extremity function after stroke. Reviews on BMT have emphasized the importance of BMT in improving upper arm functions in patients with chronic stroke $[1,2]$. BMT induces a short-term increase in corticospinal excitability after training [3, 4].

However, because of the requirement of the ability to voluntarily move the paretic limb, BMT is not indicated for patients with severe paresis. Moreover, the effectiveness of BMT is limited when patients have severe upper arm paresis as the effectiveness of the intervention depends on the level of severity [4].

For these patients, several technologies producing artificial movement of the paretic limb may complement difficulties in performing BMT. Electrical stimulation of peripheral nerves innervating muscles is one of the methods for easily producing artificial movement. Methods for triggering contralateral limb movement have been used as methods to assist BMT. Knutson et al. developed a protocol for contralaterally controlled functional electrical stimulation (CCFES) and investigated the effectiveness of improving the hand function of stroke patients [5]. Furthermore, CCFES in patients with hemiparesis found a significant reduction in hand impairment after training sessions [6]. However, previous studies have used electric stimulation with voluntary contraction of the paretic arm. Therefore, it is not clear whether artificial symmetrical movement has the potential to change the cortical excitability of the resting hemisphere. Another way to create artificial movements is transcranial magnetic stimulation (TMS) over the motor cortex [7]. Some studies have used voluntary contraction or movementtriggered stimulation methods for TMS timing [8, 9]. However, the effects of artificial symmetrical movement on cortical excitability through TMS have not been reported.

Therefore, in the present study, we compared the cortical excitability on the ipsilateral side of voluntary movements with the conventional BMT and artificial symmetrical movements generated by FES and TMS in healthy individuals.

\section{Methods}

\section{Participants}

Experiments were performed on 12 right-handed healthy adult participants ( 8 men and 4 women; age range, $20-50$ years; mean age, $26 \pm 8$ years) without any neurological diseases after obtaining written informed consent. Ethical approval was obtained from the ethics review committee of the School of Rehabilitation Sciences, Health Sciences University of Hokkaido (approval number: 18R057066).

Participants were seated on a chair comfortably, and the forearms and wrists on both sides were fixed on a table in a neutral position during the experiments.

General experimental protocol

The general experimental conditions and time course in the present study are illustrated in Fig. 1. This study was performed with a counterbalanced crossover design, which consisted of three experimental 
sessions with different interventions: (1) bilateral finger movement training (BFT) involving bilateral thumb abduction, (2) electromyographic (EMG) activity of the right abductor pollicis brevis (APB)triggered TMS of the ipsilateral M1 (APB-triggered i-TMS), and (3) EMG activity of the right APB-triggered contralateral median nerve stimulation (APB-triggered c-MNS) (Fig. 1A). The intervention consisted of two blocks that lasted for 15 min each (see below for details). A break period of 5 min was interposed between blocks. Outcome measurements were performed before (baseline), immediately after (at $0 \mathrm{~min}$ ), and at 20, 40, and 60 min after the intervention. Each session lasted for $\sim 2$ hours and was performed on a separate day with at least one week gap between sessions.

\section{Recording}

Surface EMGs were recorded bilaterally from the APB. A pair of $\mathrm{Ag} / \mathrm{AgCl}$ disc electrodes (NE-101; Nihon Kohden, Tokyo, Japan) were placed with the active electrode over the muscle belly and the reference electrode over the metacarpophalangeal joint of the thumb. The EMG signals were amplified $(\times 1,000)$ and band-pass filtered $(5-3,000 \mathrm{~Hz}$ ) with a bioamplifier (BIOTOP 6R12; NEC San-ei Instruments, Tokyo, Japan). The analog EMG signals were digitized at $6 \mathrm{kHz}$ and stored on a computer using an A/D converter (Power1401-3; Cambridge Electronic Design, Cambridge, UK) and data acquisition software (Spike2 version 7; Cambridge Electronic Design).

\section{TMS}

A monophasic single-pulse TMS was administered using a magnetic stimulator (Magstim 2002; Magstim, Whitland, UK) and a figure-of-eight coil (D70 Alpha B.I.; Magstim). For the paired-pulse TMS protocol, a set of two magnetic stimulator units through a BiStim connecting module (Magstim BiStim²; Magstim) was used. The coil was held over the right scalp so that the induced current flowed in the posterioranterior direction in the brain $[10,11]$. The optimal coil position to produce a large MEP in the left APB was determined at rest and was marked on the scalp prior to data collection. The resting motor threshold (RMT) was defined as the minimum intensity that produced an MEP of $>50 \mu \mathrm{V}$ in the left APB in at least 5 of 10 consecutive TMS pulses at $0.2 \mathrm{~Hz}$ while participants were at rest. The RMT was determined by increasing or decreasing the stimulus intensity in steps of $1 \%$ of the maximum stimulator output.

Median nerve stimulation (MNS)

A single rectangular electrical pulse (1-ms duration) was administered with an electrical stimulator (SEN8203; Nihon Kohden) connected to a constant-voltage isolator unit (SS-104J; Nihon Kohden). The left median nerve at the wrist was stimulated using a pair of surface electrodes (NE-101; Nihon Kohden) with a bipolar montage ( $2 \mathrm{~cm}$ apart, cathode on the proximal). The optimal electrode positions to elicit a large motor (M-) wave in the left APB were determined, and the electrodes were fixed with elastic surgical tape.

Interventions 
Three interventional protocols are described in Fig. 1A. As mentioned above, each intervention included two blocks of 180 trials (360 trials in total). In the BFT protocol, participants were requested to perform ballistic voluntary abduction movements of both thumbs simultaneously with maximum effort in response to an auditory imperative cue (tone burst, $2 \mathrm{kHz}, 100-\mathrm{ms}$ duration). A warning cue (tone burst, $1 \mathrm{kHz}, 100-\mathrm{ms}$ duration) was presented at $0.8-1.5 \mathrm{~s}$ prior to the imperative cue to maintain arousal. The set of warning and imperative cues was presented at an interval of $5 \mathrm{~s}$. Visual feedback of the rectified and smoothed EMG signals was given on a monitor in front of the participants to maintain the EMG activity during movements. In the APB-triggered i-TMS and APB-triggered c-MNS protocols, participants were asked to perform a ballistic voluntary abduction movement of the right thumb alone and to keep the left APB relaxed during the intervention. In these protocols, a specific EMG waveform of the right APB was discriminated in real time by a template-matching algorithm of a spike detector (Alpha Spike Detector; Alpha-Omega Engineering, Nazareth, Israel) and converted into a transistor-transistor logic (TTL) pulse event. Only a single-pulse event was generated per movement to prevent unnecessary high-frequency stimulation, which would cause muscle fatigue or other undesired effects. Then, the generated TTL pulse triggered TMS over the right M1 in the APB-triggered i-TMS and electrical stimulation of the left median nerve in the APB-triggered c-MNS. The stimulus intensity of TMS was set at $120 \%$ of the RMT. The stimulus intensity of MNS was set at $120 \%$ of the stimulator output that was required to elicit the maximum M-wave.

\section{Outcome measurements}

Four different outcomes were measured; RMT, MEP amplitudes, short-interval intracortical inhibition (SICI), and intracortical facilitation (ICF). All measurements were performed at rest. To measure MEP amplitude, 10 MEPs were evoked by a train of single TMS pulses at $0.17-0.25 \mathrm{~Hz}$. The TMS intensity that elicited an MEP of $0.5-1 \mathrm{mV}$ was determined at baseline, and the intensity was kept constant across time periods. For measurements of SICI and ICF, a test-conditioning stimulation paradigm was used. Ten test MEPs and 10 conditioned MEPs were elicited by randomly altered single (for test MEPs) and paired (for conditioned MEPs) TMS pulses at $0.17-0.25 \mathrm{~Hz}$. The stimulus intensity of the test TMS pulse was set at an intensity that evoked an MEP of $1 \mathrm{mV}$ and adjusted at each time period if necessary. The stimulus intensity of the conditioning TMS pulse was set at 0.8 times the RMT [12]. Interstimulus intervals between the test and conditioning pulses were set at $2 \mathrm{~ms}$ for $\mathrm{SICl}$ and $10 \mathrm{~ms}$ for ICF.

In the offline analysis, the peak-to-peak amplitude of the MEP was measured in individual unrectified EMG sweeps. The mean value of the peak-to-peak amplitude across 10 sweeps was then calculated. The MEP amplitude for single-pulse TMS was normalized by the baseline value. For SICI and ICF, the amplitude of the conditioned MEP was normalized by that of the MEP test.

\section{Statistics}

All statistical tests were performed using SPSS software (SPSS Statistics version 25; IBM, Chicago, IL, USA). A two-way repeated measures analysis of variance (ANOVA) (intervention $\times$ time) was performed for each dependent variable. If the reported $F$ value was statistically significant, a post-hoc test was 
performed with Dunnett's test to reveal differences from the baseline values. A $p$ value $<0.05$ was considered statistically significant. Group data are shown as the mean \pm standard error of the mean.

\section{Results}

RMT and MEP amplitude

The averaged data of the RMT values are shown in Fig. 2A. A two-way ANOVA showed no significant effect of intervention $[\mathrm{F}(2,22)=2.79, p=0.08]$ and time $[\mathrm{F}(4,44)=0.94, p=0.45]$ and interaction $[\mathrm{F}(8$, $88)=0.84, p=0.57]$. Changes in the mean MEP amplitude are presented in Fig. 2B. Two-way ANOVA showed a significant effect of time $[F(4,44)=17.39, p<0.01]$, but no effect of intervention $[F(2,22)=$ $1.09, p=0.35)$ and interaction $[F(8,88)=1.69, p=0.11]$. Post-hoc analysis with Dunnett's test indicated that the MEP amplitude significantly increased at 0,20 , and 40 min post-intervention in BFT $(p<0.05)$; at $0,20,40$, and 60 min post-intervention in APB-triggered i-TMS $(p<0.05)$; and at 20 and 40 min postintervention in APB-triggered c-MNS $(p<0.05)$ in comparison to the baseline values. For the MEP amplitude at baseline, separate one-way ANOVA revealed no effect of intervention $[F(2,22)=3.28, p=$ 0.06].

\section{$\mathrm{SICl}$}

The SICl group data are shown in Fig. 3A. Two-way ANOVA yielded a significant effect of time $[\mathrm{F}(4,44)=$ $3.84, p=0.009]$, but no effect of intervention $[F(2,22)=0.03, p=0.98]$ and interaction $[F(8,88)=1.82, p=$ $0.08]$. The results of the post-hoc test showed that $\mathrm{SICI}$ was significantly decreased at 0 min postintervention in $\mathrm{BFT}(p=0.01)$ and APB-triggered i-TMS protocols $(p=0.01)$ when compared to baseline values. There was no significant difference in $\mathrm{SICl}$ at different time points in the APB-triggered c-MNS protocol $(p=0.61)$.

\section{ICF}

Changes in ICF are shown in Fig. 3B. Two-way ANOVA revealed a significant effect of time $[F(4,44)=$ $6.13, p=0.01]$, but no effect of intervention $[\mathrm{F}(2,22)=0.66, p=0.57]$ and interaction $[\mathrm{F}(8,88)=1.06, p=$ 0.09 ]. Post-hoc test results showed that ICF was significantly increased at 0 min post-intervention in BFT $(p=0.02)$ and 20 min post-intervention in the APB-triggered c-MNS protocol $(p=0.001)$. In the APBtriggered i-TMS protocol, there was no significant difference in ICF at different time periods when compared to the baseline values $(p=0.09)$.

\section{Discussion}

In this study, we found that the MEP amplitude increased after all tested protocols using symmetrical mirror movements; BFT is considered the normal protocol, the APB-triggered c-MNS protocol uses the MNS, and the APB-triggered i-TMS protocol uses TMS. Our results suggest that despite being voluntarily 
or artificially caused, repetitive symmetrical mirror movements induce short-term modification of the motor cortical and corticospinal excitabilities.

In the present study, the increase in MEP amplitude lasted up to 40 to 60 min in each intervention. Numerous studies have demonstrated that short-term changes in the MEP can be induced by neuromodulation protocols such as repetitive TMS (rTMS), transcranial direct current stimulation (tDCS), and paired associative stimulation (PAS). For instance, low-frequency rTMS causes about 60 min of cortical depression, and high-frequency rTMS promotes cortical activity over time. These changes are similar to the cell-level experiments that revealed long term depression (LTD) and long term potentiation (LTP) [13]. The tDCS protocol, which generates a small amount of continuously flowing direct current over the scalp, is also one of the technologies that induce plasticity. The tDCS protocols showed increased cortical excitability, which lasted for about 30 min after stimulation [14]. PAS, which uses low-frequency nerve stimulation and TMS over the optimal cranial site to stimulate the target muscle, is a neuromodulation technique that causes LTP-like plasticity. The PAS protocol increased the MEP amplitude by about $150 \%$ and lasted over 30 min [15]. Likewise, the long-lasting increase in MEP could be induced by the present protocols, but the duration of the effects seems to be relatively long compared with that of the previous neuromodulation protocols.

It is also known that cortical excitability changes can occur with electrical stimulation of the peripheral nerves and rTMS alone. Previous studies have demonstrated that cortical plasticity can be induced by electric stimulation of peripheral nerves [16]. However, most of the previous studies using electrical stimulation used high-frequency stimulation or train pulse stimulation. According to a review by Carson and Buick (2019), the typical frequency of neuromuscular electrical stimulation (NMES) to activate sensory and motor axons should be $1-100 \mathrm{~Hz}$ [17]. Upon comparison with these studies, it was considered that the number of stimulation pulses in our protocol was too small to cause an impact on the brain.

As mentioned previously, it is widely known that rTMS causes brain plasticity. In particular, low-frequency rTMS causes depression of cortical activity [18]. The stimulation frequency used was $0.2 \mathrm{~Hz}$, and if the effect was due to rTMS, MEP should be decreased. However, our results showed increased MEP. Therefore, we speculate that a synergistic effect was observed when combining the input by interhemispheric communication from the contralateral motor cortex with the input via the sensory cortex by peripheral electrical stimulation or direct cortical stimulation by TMS.

The increase in MEP amplitude may occur because of the interactions in the motor cortex or subcortical structures [19]. The MEP may be increased because of primary mechanisms that increase the facilitatory circuits and/or decrease the inhibitory circuits in the M1. The RMT is one of the factors that change MEP, which reflects the stimulus intensity needed to activate the most excitable corticospinal neurons and motoneurons [20]. In our study, RMT did not change throughout the experimental protocols. Hence, the influences of these elements are likely small. In our BFT protocol, the ICF was significantly increased, and the SICI was decreased. According to Waller et al. (2008), bilateral movement caused increased ICF and 
reduced ICl in both hemispheres [21]. These results are consistent with those of our study, and the voluntary mirror-symmetrical movement was thought to induce enhancement of the facilitation circuit and attenuation of the inhibitory circuit. In the APB-triggered c-MNS protocol, ICF significantly increased, but $\mathrm{SICl}$ did not show a significant change. Conversely, the APB-triggered i-TMS protocol did not show a significant change in ICF, but SICl significantly decreased. Based on this, it is considered that the function of reducing the inhibitory circuit in APB-triggered i-TMS and enhancing the facilitatory circuits in the APBtriggered c-MNS protocol contributed to the increase in MEP. There are no robust effects such as in the BFT, but changes in the cortical circuits may have occurred in both protocols. The long-lasting effect has been thought to be the evidence of LTP-like plasticity [15]. Additionally, they discussed the mechanics of plastic change as the spike-timing-dependent plasticity (STDP), which was investigated in a cell study [22]. There are some similarities to our protocols that use inputs from multiple paths for a specific neuron. However, the present study cannot precisely explain why these results occur as our protocol could not precisely control the interstimulus interval, as in the PAS protocol.

\section{Conclusions}

In conclusion, the BFT, APB-triggered i-TMS, and APB-triggered c-MNS protocols increased the MEP amplitude after intervention. Thus, voluntarily or artificially caused symmetrical mirror movements enhance corticospinal excitability. However, the changes in the SICI and ICF differed depending on the protocol. These differences may need to be taken into account when applying these protocols to rehabilitation such as that after stroke.

\section{Abbreviations}

APB

Abductor pollicis brevis muscle

BFT

Bilateral finger training

BMT

Bilateral motor training

EMG

Electromyographic

FES

Functional electrical stimulation

ICF

Intracortical facilitation

M1

Primary motor cortex

MEP

Motor-evoked potential 
MNS

Median nerve stimulation

RMT

Resting motor threshold

rTMS

Repetitive transcranial magnetic stimulation

SICl

Short-interval intracortical inhibition

TMS

Transcranial magnetic stimulation

\section{Declarations}

- Ethics approval and consent to participate: This study was approved by the ethics review committee of the School of Rehabilitation Sciences, Health Sciences University of Hokkaido. Written informed consent was obtained from the participants after providing the necessary information before participation.

- Consent for publication: Not applicable.

- Availability of data and materials: The datasets used and/or analyzed during the current study are available from the corresponding author upon reasonable request.

- Competing interests: The authors declare that they have no competing interests.

- Funding: Financial support for the study was received from JSPS KAKENHI (16K01513).

- Author contributions: YS, the corresponding author, was involved in designing the study, analysis and interpretation of data, and drafting of the final manuscript. PK participated in collecting data from the participants, analysis of data, and drafting of the manuscript. SS participated in designing the study, data collection, analysis and interpretation of data, and drafting of the manuscript. All authors participated in the discussion and provided inputs to improve the content of the manuscript. All authors read and approved the final manuscript.

- Acknowledgments: We would like to acknowledge all participants who voluntarily participated in this study.

\section{References}

1. Cauraugh JH, Summers JJ. Neural plasticity and bilateral movements: a rehabilitation approach for chronic stroke. Prog neurobiol. 2005;75(5):309-20.

2. Stewart KC, Cauraugh JH, Summers JJ. Bilateral movement training and stroke rehabilitation: a systematic review and meta-analysis. J Neurol Sci. 2006;244(1-2):89-95.

3. Byblow WD, Stinear CM, Smith MC, Bjerre L, Flaskager BK, McCambridge AB. Mirror symmetric bimanual movement priming can increase corticomotor excitability and enhance motor learning. 
PLoS One. 2012;7(3):e33882.

4. Waller SM, Whitall J. Bilateral arm training: why and who benefits? NeuroRehabilitation. 2008;23(1):29-41.

5. Knutson JS, Harley MY, Hisel TZ, Chae J. Improving hand function in stroke survivors: a pilot study of contralaterally controlled functional electric stimulation in chronic hemiplegia. Arch Phys Med Rehabil. 2007;88(4):513-20.

6. Knutson JS, Hisel TZ, Harley MY, Chae J. A novel functional electrical stimulation treatment for recovery of hand function in hemiplegia: 12-week pilot study. Neurorehabil Neural Repair. 2009;23(1):17-25.

7. Ridding MC, Rothwell JC. Is there a future for therapeutic use of transcranial magnetic stimulation? Nat Rev Neurosci. 2007;8(7):559-67.

8. Edwardson MA, Avery DH, Fetz EE. Volitional muscle activity paired with transcranial magnetic stimulation increases corticospinal excitability. Front Neurosci. 2015;8:442.

9. Thabit MN, Ueki Y, Koganemaru S, Fawi G, Fukuyama H, Mima T. Movement-related cortical stimulation can induce human motor plasticity. J Neurosci. 2010;30(34):11529-36.

10. Werhahn KJ, Fong JK, Meyer BU, Priori A, Rothwell JC, Day BL, Thompson PD. The effect of magnetic coil orientation on the latency of surface EMG and single motor unit responses in the first dorsal interosseous muscle. Electroencephalogr Clin Neurophysiol/Evoked Potentials Sect. 1994;93(2):138-46.

11. Sakai K, Ugawa Y, Terao Y, Hanajima R, Furubayashi T, Kanazawa I. Preferential activation of different I waves by transcranial magnetic stimulation with a figure-of-eight-shaped coil. Exp Brain Res. 1997;113(1):24-32.

12. Kujirai T. Caramia MD, Rothwell JC, Day BL, Thompson PD, Ferbert A, Wroe S, Asselman P. Corticocortical inhibition in human motor cortex. J Physiol. 1993;471:501-14.

13. Klomjai W, Katz R, Lackmy-Vallée A. Basic principles of transcranial magnetic stimulation (TMS) and repetitive TMS (rTMS). Ann Phys Rehabil Med. 2015;58(4):208-13.

14. Desmond A, Mohsen MS, Min-Fang K, Michael AN. Induction of Iong-term potentiation-like plasticity in the primary motor cortex with repeated anodal transcranial direct current stimulation-Better effects with intensified protocols?. Brain Stimul. 2020.

15. Stefan K, Kunesch E, Cohen LG, Benecke R, Classen J. Induction of plasticity in the human motor cortex by paired associative stimulation. Brain. 2000;123(3):572-84.

16. Chipchase LS, Schabrun SM, Hodges PW. Peripheral electrical stimulation to induce cortical plasticity: a systematic review of stimulus parameters. Clin Neurophysiol. 2011;122(3):456-63.

17. Carson RG, Buick AR. Neuromuscular electrical stimulation-promoted plasticity of the human brain. J Physiol. 2019.

18. Chen RM, Classen J, Gerloff C, Celnik P, Wassermann EM, Hallett M, Cohen LG. Depression of motor cortex excitability by low-frequency transcranial magnetic stimulation. Neurology. 1997;48(5):1398- 
403.

19. Ziemann UL, Rothwell JC, Ridding MC. Interaction between intracortical inhibition and facilitation in human motor cortex. J Physiol. 1996;496(3):873-81.

20. Rossini PM, Burke D, Chen R, Cohen LG, Daskalakis Z, Di lorio R, Di Lazzaro V, Ferreri F, Fitzgerald PB, George MS, Hallett M. Non-invasive electrical and magnetic stimulation of the brain, spinal cord, roots and peripheral nerves: basic principles and procedures for routine clinical and research application. An updated report from an IFCN Committee. Clin Neurophysiol. 2015;126(6):1071-107.

21. Waller SM, Forrester L, Villagra F, Whitall J. Intracortical inhibition and facilitation with unilateral dominant, unilateral nondominant and bilateral movement tasks in left-and right-handed adults. $J$ Neurol Sci. 2008;269(1-2):96-104.

22. Karmarkar UR, Najarian MT, Buonomano DV. Mechanisms and significance of spike-timing dependent plasticity. Biol Cybern. 2002;87(5-6):373-82.

\section{Figures}

A

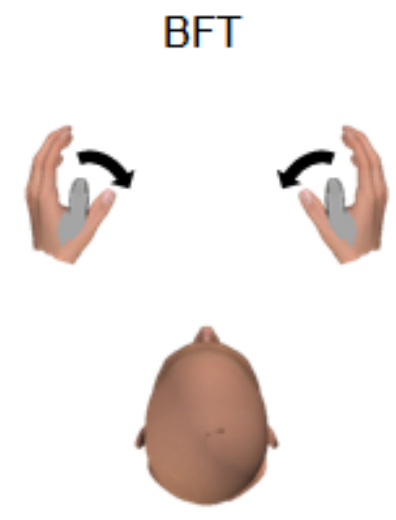

APB-triggered cMNS
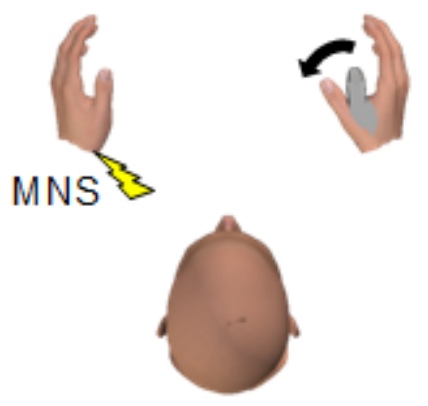

APB-triggered
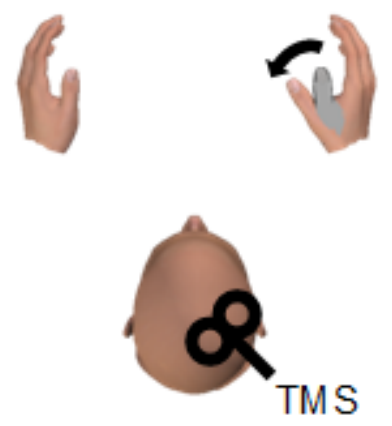

B

Baseline Intervention $\quad 0 \quad 20 \quad 40 \quad 60 \mathrm{~min}$

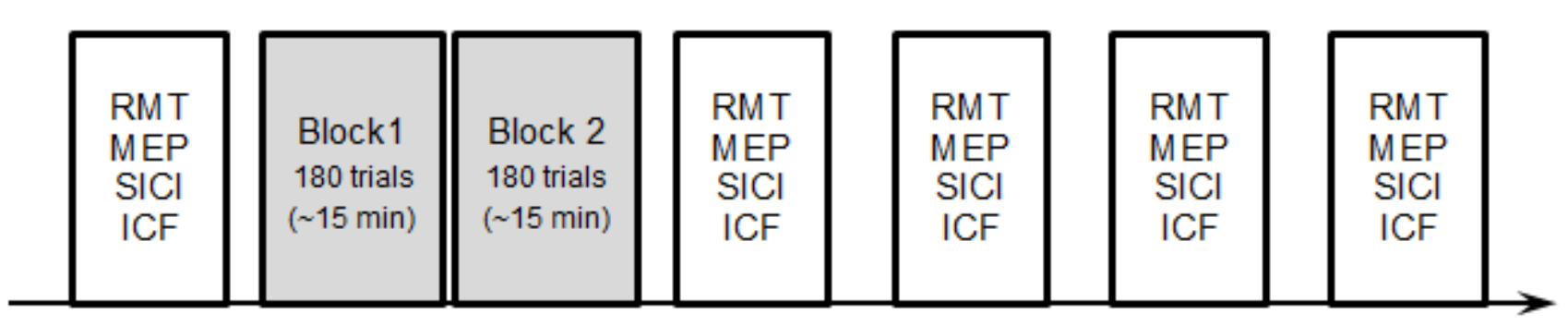

Figure 1 
Protocol of the experiment A: Schematic illustration of the intervention protocols used in the present study. Left panel: Bilateral finger training (BFT) session and bilateral thumb abduction. Middle panel: In the electromyogram (EMG)-triggered contralateral median nerve stimulation (APB-triggered c-MNS) session, participants with unilateral thumb abduction and contralateral MNS were triggered by the EMG activity of the ipsilateral abductor pollicis brevis (APB) muscle. Right panel: In the EMG-triggered ipsilateral transcranial magnetic stimulation (APB-triggered i-TMS) session, participants exerted unilateral thumb abduction, and TMS over the motor cortex was triggered by the EMG activity of the ipsilateral APB B: Time course of each experiment session. Four types of outcome measurements [resting motor threshold (RMT), motor-evoked potential (MEP), short-interval intracortical inhibition (SICI), and intracortical facilitation (ICF)] were performed before (baseline), immediately after (0), and 10, 20, 40, and 60 min after the intervention. The intervention period consisted of two blocks ( 180 trials over $\sim 15$ min each) 


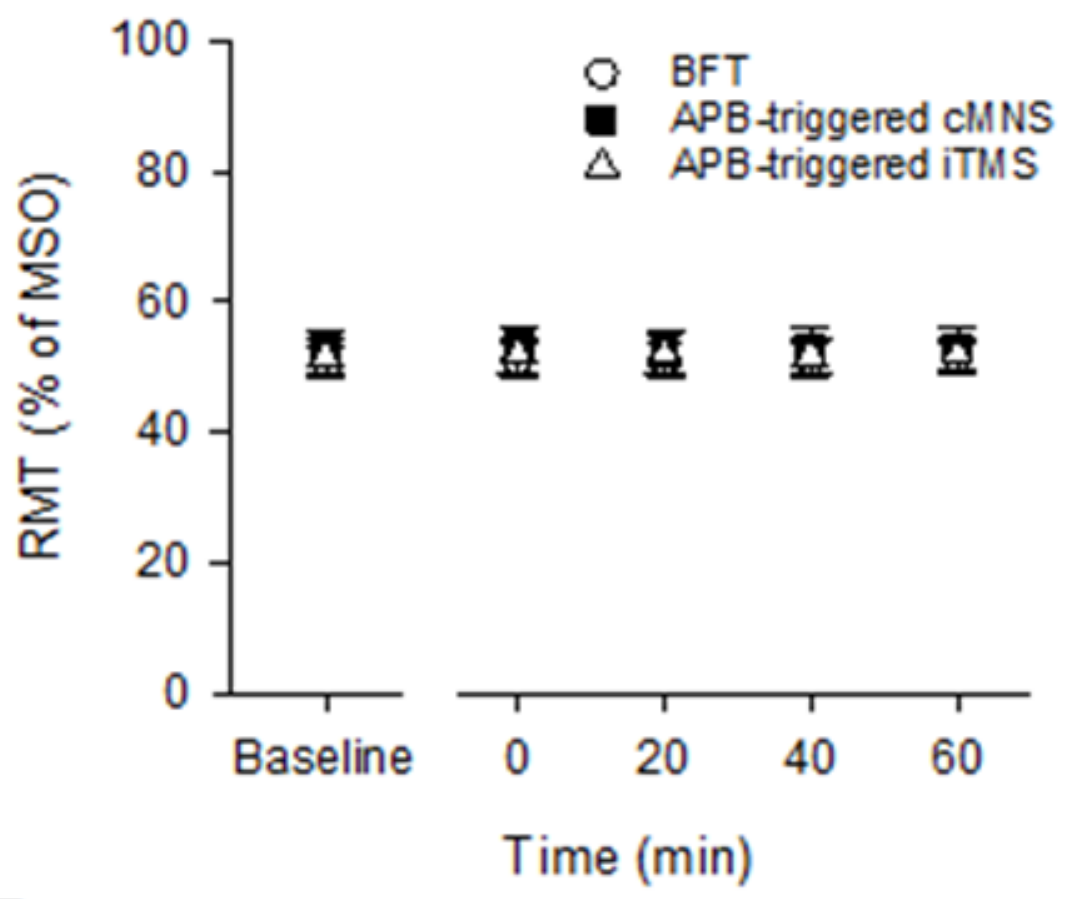

B

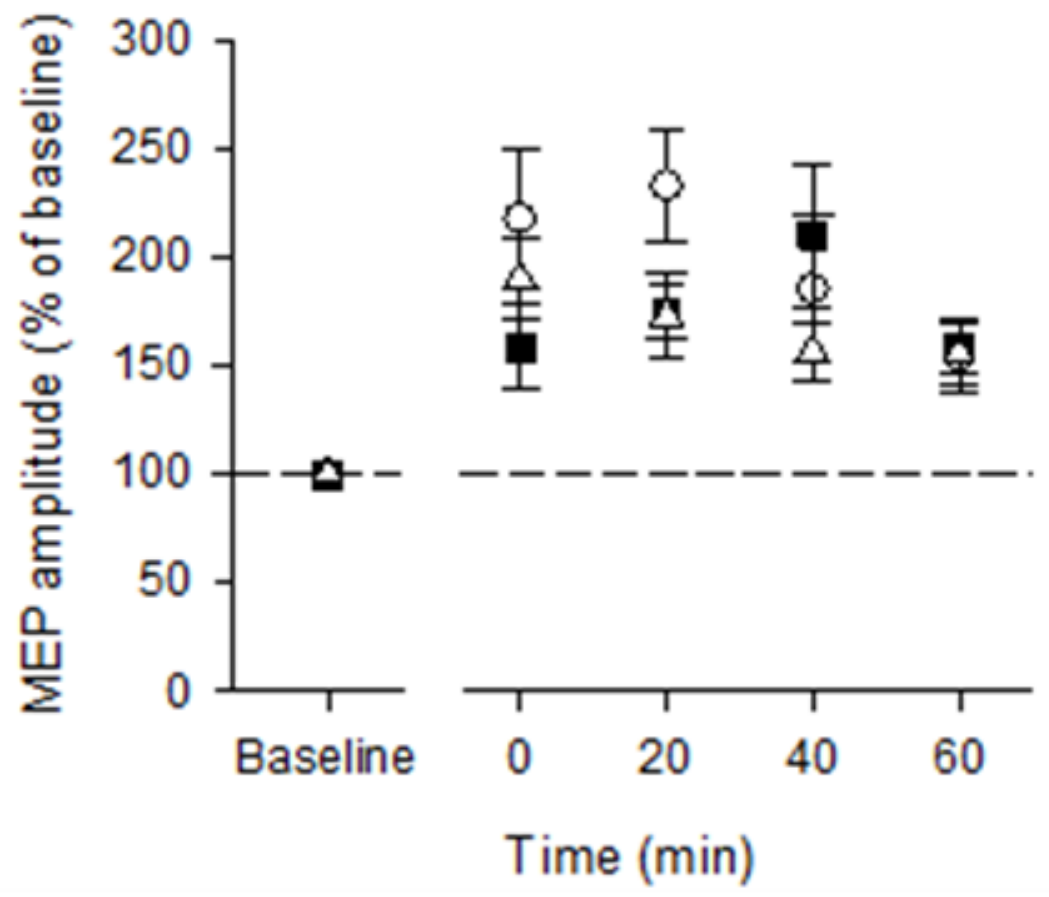

Figure 2

Averaged data of resting motor threshold (RMT) (A) and amplitude of the motor-evoked potential (MEP) (B) in all participants The RMT is expressed as percent of the maximum stimulator output (MSO). The MEP amplitude was expressed as percent of the baseline value. Each plot and error bar represent the mean and standard error of the mean, respectively 
A $\quad$ B
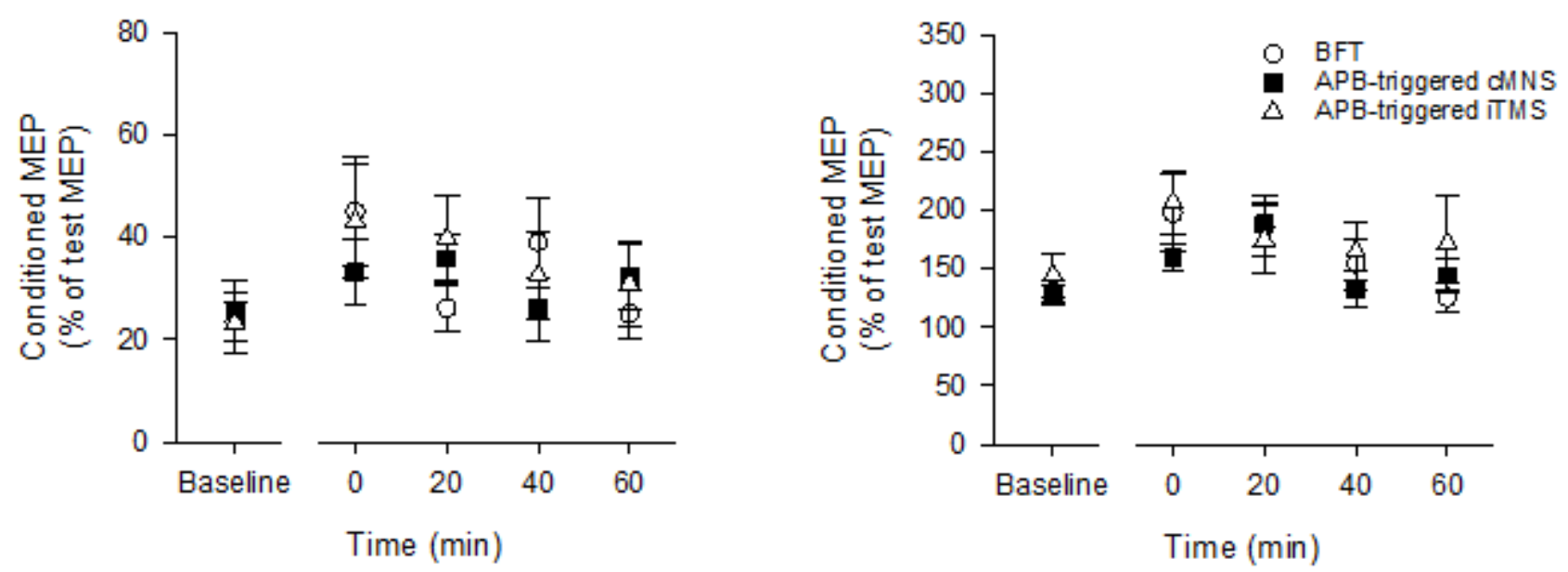

\section{Figure 3}

Population data of short-interval intracortical inhibition (SICI) (A) and intracortical facilitation (ICF) (B) in all participants The conditioned MEP amplitude is expressed as percent of the unconditioned MEP amplitude. Each plot and error bar represent the mean and standard error of the mean, respectively 